\section{Beating every challenge}

Dental professionals looking for an intraoral scanner that can really go the extra mile and overcome any challenge should choose the CS 3600 intraoral scanner from Carestream Dental.

Seamlessly merging conventional impressions with digital scans, the scanner implements a hybrid scan workflow that can identify even the most challenging margin lines.

Furthermore, the CS 3600 offers continuous scanning to provide professionals with better data to work from, which results in better-fitting prosthetics for the patient.

For more information, contact Carestream Dental on 0800 1699692 or visit www.carestreamdental.co.uk.

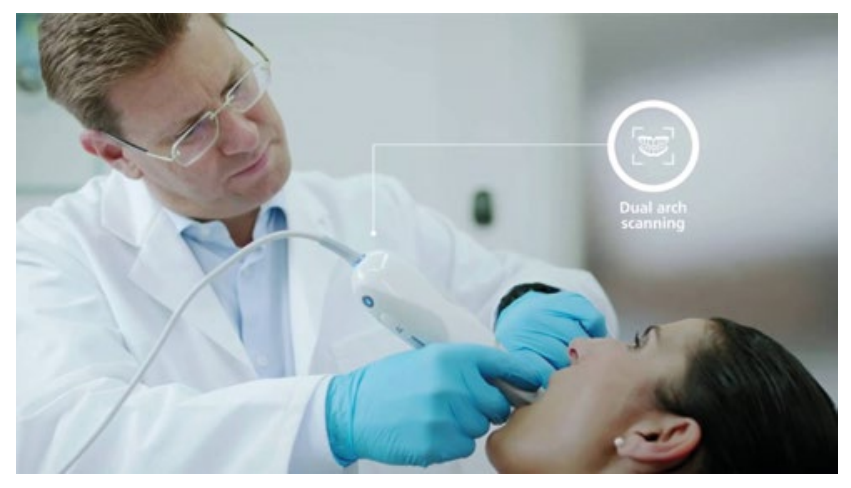

\section{Screening preference}

Dental Advisor have rated Goccles - the oral cancer screening glasses - as their preferred product for oral cancer screening.

The organisation provides dental professionals worldwide with concise, accurate, and objective information on a product's performance, combining clinical experience with laboratory data and long-term in-vivo performance of materials over time.

With an enviable rating of $92 \%$, they found the Goccles system easy to use and effective at displaying early lesions.

Several of the consultants who reviewed the product also commented on its appeal to patients, with one saying 'Patients liked that it was obvious that I was giving them an oral cancer screening and using technology. Patients perceived that they were getting a better-quality exam.'

Goccles allow the clinician to run a simple, non-invasive and painless test using the technology of fluorescence and cell-tissue autofluorescence by utilising the wavelengths emitted by curing-lights.

This allows the user to see in a clear and accurate way any anomalies of the oral cavity. The basic principle is that the auto-fluorescence of abnormal cells lining the mouth when exposed to light, differs to that seen occurring in normal cells.

Goccles glasses allow the clinician to see differences in auto-fluorescence of the tissues, with normal cells appearing green and abnormal cells dark. Goccles are distributed exclusively through Dental Sky.

More information is available by visiting https://www. dentalsky.com/goccles-oral-cancer-screening-glasses.html.

\section{T-scan or not to scan}

Enhance your diagnostic capabilities with the innovative T-scan occlusal analyser.

This state-of-the-art device features a unique sensor that patients bite down on in order to capture highly detailed and accurate occlusal data for immediate chairside review.

With the T-scan, practitioners can perform effective diagnostics that enable patients to leave the practice with a well-balanced and comfortable occlusion. As a result, undue stress on restorations and cusps is reduced, thereby enabling clinicians to maximise on valuable chair time and minimise the risk of return visits due to prosthetic complications.

More information is available by calling Clark Dental on 01270613750 , emailing info@clarkdentalsales.co.uk, or visiting www.tekscan.com.

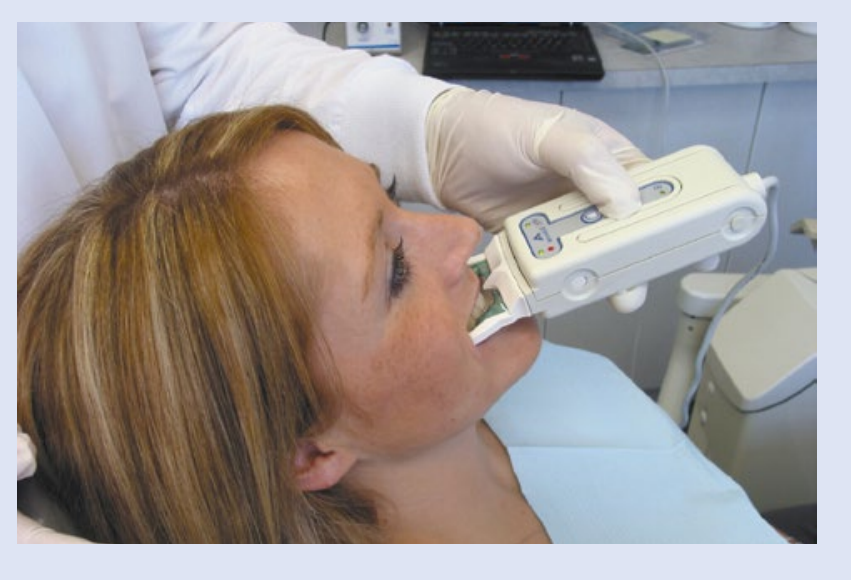

\section{Looking for an efficient, hassle-free sale?}

Any dental professional looking for an efficient and hassle-free sale should look no further than Rodericks Dental.

You will work with an experienced team that understands the challenges of selling a practice, as well as how to overcome them.

Able to complete in as little as 12 weeks from signing Heads of Terms, visit your practice outside normal opening hours and contribute towards legal fees when a recommended representative is used, Rodericks aims to make the process as easy as possible.

In addition, the group's commitment to excellent patient care and professional career development will ensure your team and patients are in safe hands.

For more information, please visit https://www.rodericksdental. co.uk/sell-your-practice/.

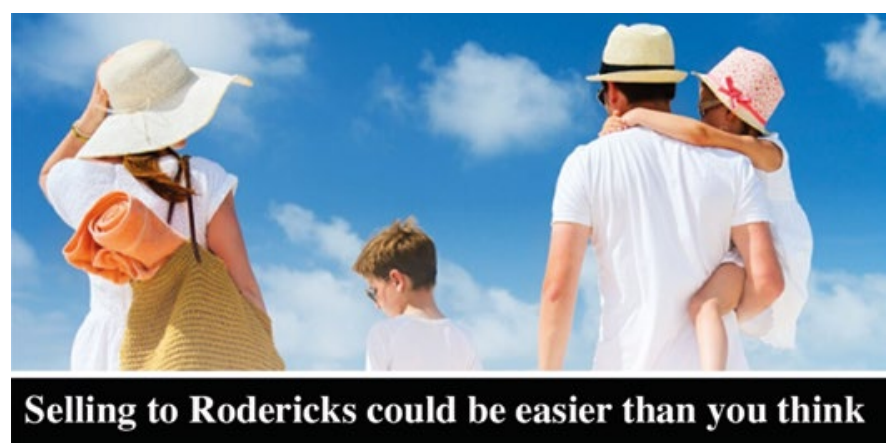

